International Journal on Intelligent Electronic Systems, Vol.3, No.1, January 2009

\title{
PERFORMANCE COMPARISON OF NON-LINEAR INTERFERENCE CANCELLATION TECHNIQUES FOR MULTIUSER DETECTION IN DS-CDMA SYSTEM
}

\author{
Lokesh Tharani ${ }^{1}$, R.P.Yadav ${ }^{2}$ \\ Department of Electronics and Communication Engineering \\ ${ }^{1} J a g a n N a t h$ Gupta Institute of Engineering and Technology, Jaipur, India \\ ${ }^{2}$ Malaviya National Institute of Technology Jaipur, India \\ E-mail: 'tharani123@gmail.com, ²rp_yadav@yahoo.com
}

\begin{abstract}
Direct sequence code division multiple access (DS-CDMA) is a most popular mobile communication technology. In DS-CDMA communication, signals of all users overlap in time and frequencies and cause multiple access interference (MAI). The conventional DS-CDMA detectors follow the single user matched filter detection technique, in which each user is detected individually without regard for other users and ignores the existence of MAI. Its performance is not very satisfactory and is particularly limited by the near-far problem. Multiuser detection is the efficient technique to use the capabilities of code-division multiple access (CDMA), which is becoming the ubiquitous air-interface in future generation communication systems. The problem of MAI is vital for a CDMA system. The different types of multiuser detectors are available to mitigate the MAl. The different sub optimal multiuser detector offers performance in between the single user matched filter and the optimal multiuser detectors with substantially reduced complexity. This paper presents the simulation results regarding performance comparison of some most popular non-linear multiuser detectors i.e., multistage detectors, decision- feedback detectors and subtractive interference cancellation.
\end{abstract}

Key words: Multiuser detection (MUD), multiple access interference (MAI), decision feedback detector, successive interference cancellation detector (SIC) and parallel interference cancellation (PIC) detector.

\section{INTRODUCTION}

The tremendous increase in demand for wireless services has caused a search for techniques to improve the capacity of current digital wireless communication. To bring this vision for future, major improvements in the current state of wireless technology are necessary. One type of wireless technology which has become very popular over the last few years is direct sequence code division multiple access (DS-CDMA). Code Division Multiple Access (CDMA) is one of the several methods of multiplexing wireless users [1]. In CDMA, users are multiplexed by distinct codes rather than by orthogonal frequency band as in frequency-division multiple accesses (FDMA), or by orthogonal time slots as in time-division multiple access (TDMA). In CDMA, all users can transmit at the same time. Also, each user is allocated the entire frequency spectrum for transmission; hence, CDMA is also known as spread spectrum communications. The DS-CDMA multiplier multiplies each user's signal by a distinct code waveform as shown in Fig. 1.

In DS-CDMA system multiple access interference (MAI) is the major factor limiting the performance and, hence, the capacity of the system. The interference from other users is known as multiple access interference (MAl). This interference is the result of random time offsets between signals, which makes it impossible to design the code waveform to be completely orthogonal. While the MAI caused by any one user is generally small, as the number of interference or their power increases, MAl becomes substantial. Therefore, analysis of the effect of MAI on the system performance [2] as well as ways to suppress MAI has been the major focus of CDMA research.

The multiuser detection is one of the best approaches to solve this problem. The code and timing (or amplitude \& phase) information of multiple users are jointly used to better detection of signal of individual user is known as multiuser detection [3]. The important assumption is that the codes of the multiple users are known to the receiver a priori. Theoretically, receivers based on multi-user detection usually outperform, but are usually more complex than receivers based on single-user detection. The applicability of multi-user receivers depends on system design issues, such as the security of joint detection, the implementation complexity, and the availability of information required performing multiuser detection. For example, let us consider a typical wireless cellular system.

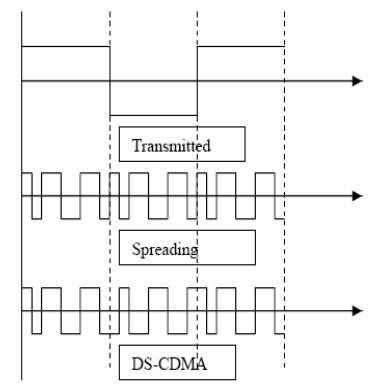

Fig.1. DS-CDMA Modulation with spreading gain $\mathrm{N}=7$ 
It would be difficult to employ multiuser receivers at the mobile units for forward-link transmission because of the limitation on the implementation complexity and the availability of information about other users. However, multiuser detection could be a viable choice in the basestation for reverse-link transmission. Verdu's seminal work published in 1986, proposed and analyzed the optimal multiuser detector that proved to be much complex for practical DS-CDMA systems. Therefore, over the last two decades or so, research has focused on finding suboptimal multiuser detector solutions, which are more feasible to implement.

Multiuser detectors [4] are classified into two categories: linear detectors and nonlinear (subtractive interference cancellation) detectors. In linear multiuser detectors, a linear mapping is applied to the soft outputs of the conventional detector to produce a new set of outputs. In nonlinear subtractive interference cancellation detection, estimates of interference are generated and subtracted out.

\section{CONVENTIONAL DS- CDMA DETECTOR}

Now-a-days single user matched filter detection technique is using in mobile communication. Although easy to implement, the performance severely degrades when the number of users goes up. The conventional detector [5] consists of a matched filter bank (a series of transversal filters in parallel), with one filter corresponding to each user. The received signal is correlated with the signature waveform of each user, and the output is used to determine the bits transmitted by each user. This detector relies on the fact that the signature sequences of any two users are close to orthogonal. It is to be noted that the matched filter receiver is not optimal (in the sense of maximizing the likelihood function) in the presence of MAI.

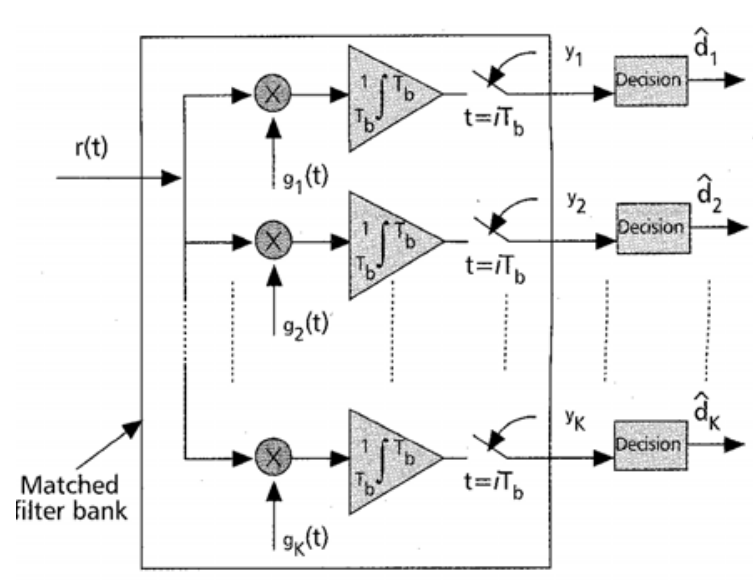

Fig. 2. The conventional DS-CDMA detector with a Matched Filter Bank
Assuming there are $\mathrm{K}$ direct-sequence users in an asynchronous single path BPSK real channel, the base band received signal can be expressed as

$$
\mathrm{r}(\mathrm{t})=\sum_{k=1}^{K} \mathrm{~A}_{k}(\mathrm{t}) \mathrm{g}_{k}(\mathrm{t}) \mathrm{d}_{k}(\mathrm{t})+\mathrm{n}(\mathrm{t})
$$

Where $A_{k}(t), g_{k}(t)$, and $d_{k}(t)$ are the amplitude, signature code waveform and modulated data of the $k$ ' th user respectively and $n(t)$ is additive white Gaussian noise (AWGN), with a two sided power spectral density of $\mathrm{N}_{0} / 2$ $\mathrm{W} / \mathrm{Hz}$. The conventional detector for received signal described in equation (1) is a bank of $\mathrm{K}$ correlators as shown in Fig.2. This figure shows that each code waveform is regenerated and correlated with the received signal in a separate detector branch of the matched filter detector. It is evident from this figure that the conventional detector follows a single user strategy, whereby each branch detects one user without regard to the existence of other users. Thus there is no sharing of multiuser

information. The success of this detector depends on the properties of the correlations between codes. We require the autocorrelation to be much larger than the crosscorrelation of different codes. The correlation value is defined as:

$$
\rho_{\mathrm{i}, k}=\frac{1}{\mathrm{~T}_{b}} \int^{T_{b}} \mathrm{~g}_{\mathrm{i}}(\mathrm{t}) \mathrm{g}_{k}(\mathrm{t}) \mathrm{dt}
$$

Here, if $\mathrm{i}=\mathrm{k}, \rho_{\mathrm{i}, \mathrm{i}}=1$, (i.e., the integral must equal one since $\left.g_{i}(t)= \pm 1\right)$ and if $i \neq k$, then $\rho_{i, k}$ between 0 to 1 . The output of the $k^{\text {th }}$ user's correlation for a particular bit interval is

$$
\begin{aligned}
\mathrm{y}_{k} & =\frac{1}{\mathrm{~T}_{b}} \int^{T_{b}} \mathrm{r}(\mathrm{t}) \mathrm{g}_{k}(\mathrm{t}) \mathrm{dt} \\
& =\mathrm{A}_{k} \mathrm{~d}_{k}+\sum_{\substack{i=1 \\
i \neq k}}^{k} \rho_{\mathrm{i}, k} \mathrm{~A}_{\mathrm{i}} \mathrm{d}_{\mathrm{i}}+\frac{1}{\mathrm{~T}_{b}} \int^{T_{b}} \mathrm{n}(\mathrm{t}) \mathrm{g}_{k}(\mathrm{t}) \mathrm{dt} \\
& =\mathrm{A}_{k} \mathrm{~d}_{k}+\mathrm{MAI}_{k}+\mathrm{Z}_{k}
\end{aligned}
$$

The correlation with the k'th user itself gives rise to the recovered data term; correlation with all the other users gives rise to multiple access interference (MAI), and correlation with the thermal noise gives the term $Z_{k}$.

\section{NEAR- FAR PROBLEM}

When the number of interfering users increasing, the amount of MAI increases. Moreover, large amplitude users further worsen the MAI of the weaker users [6], as 
can be seen by equation (3). When the received powers of all users are the same and the set of spreading sequences are properly chosen, the degradation in SNR is relatively small, if there are a moderate number of users. However, when the received powers of some of the interferers are much larger than that of the desired user, the performance degradation is large. In the context of wireless communications, this situation occurs when some of the interferers are located close to the base station while the desired user is far away.

\section{MATHEMATICAL MODEL}

In discussing multiuser detection, it is convenient to introduce a mathematical matrix vector model to describe the output of the conventional detector. To simplify our assumption, let us consider a three user system. From equation (3), the output for each of the users for one bit is

$$
\begin{aligned}
& y_{1}=A_{1} d_{1}+\rho_{2,1} A_{2} d_{2}+\rho_{3,1} A_{3} d_{3}+z_{1} \\
& y_{2}=\rho_{1,2} A_{1} d_{1}+A_{2} d_{2}+\rho_{3,2} A_{3} d_{3}+z_{2} \\
& y_{3}=\rho_{1,3} A_{1} d_{1}+\rho_{2,3} A_{2} d_{2}+A_{3} d_{3}+z_{3}
\end{aligned}
$$

This can be written in matrix-vector notation as shown in equation (5) and (6)

$$
\begin{aligned}
& {\left[\begin{array}{l}
y_{1} \\
y_{2} \\
y_{3}
\end{array}\right]=\left[\begin{array}{ccc}
1 & \rho_{2,1} & \rho_{3,1} \\
\rho_{1,2} & 1 & \rho_{3,2} \\
\rho_{1,3} & \rho_{2,3} & 1
\end{array}\right]\left[\begin{array}{ccc}
A_{1} & 0 & 0 \\
0 & A_{2} & 0 \\
0 & 0 & A_{3}
\end{array}\right]\left[\begin{array}{l}
d_{1} \\
d_{2} \\
d_{3}
\end{array}\right]+\left[\begin{array}{l}
z_{1} \\
z_{2} \\
z_{3}
\end{array}\right]} \\
& \text { Or } \\
& \quad Y=\text { RAd }+z
\end{aligned}
$$

Extending this system to a $\mathrm{K}$ user system, the vectors $\mathrm{d}, \mathrm{y}$ and $\mathrm{z}$ are $\mathrm{K}$-vectors that hold data, matched filter outputs and noise of all $\mathrm{K}$ users, respectively. The matrix $A$ is a diagonal matrix containing the corresponding received amplitudes, the matrix $R$ is a $\mathrm{K} \times \mathrm{K}$ correlation matrix, whose entries contain the values of the correlation between every pair of codes. Note that $\rho_{i, k}=\rho_{k, i}$, the matrix R is then a symmetric matrix. Matrix $\mathrm{R}$ can be broken into matrices, one representing the autocorrelation and the other the crosscorrealtions. Therefore equation (3) can be expressed as

$y=A d+Q A d+z$

Where $Q$ contains the off-diagonal (crosscorrealtions of $R$ ), that is $R=I+Q$, (I is the Identity Matrix). The first term Ad is the decoupled data weighted by the received amplitudes. The second term QAd represents the MAl interference.

\section{NON-LINEAR MULTIUSER DETECTORS}

These are upcoming future technologies for CDMA mobile communication. The basic principle underlying these detectors is the creation at the receiver of separate estimates of the MAI contributed by each user in order to subtract out some or all of the MAI seen by each user. Such detectors are often implemented with multiple stages, where the expectation is that the decisions will improve at the output of successive stages. These algorithms [4] can divide in three categories:

A. Multistage detectors.

B. Decision-feedback detectors.

C. Subtractive interference cancellation.

The first two classes of algorithms are decision directed. They utilized previously made decisions of other users to cancel interference present in the signal of the desired user. These algorithms require estimation of channel parameter and coherent detection. The third type of algorithm can use soft algorithms require estimation of channel parameters and coherent detection. The third type of algorithm can use soft decisions rather than hard decision to remove MAI components.

\section{A. Multistage detectors}

In this detector, interferences are cancelled out in different stages. The n'th stage of this detector uses decisions of the (n-1)'th stage to cancel MAI present in the received signal. Thus, maximization is over one bit a time, instead of over $k$ bits. The Fig. 3 shows this concept.

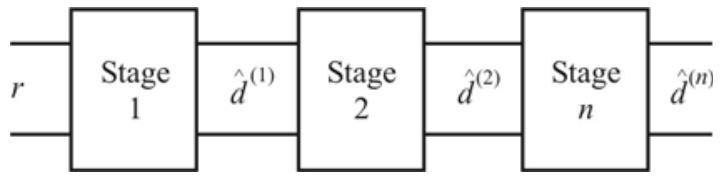

Fig. 3. The multistage detector

$$
\begin{aligned}
& \hat{d}_{1}^{(1)}=\operatorname{Sgn}\left(r_{1} \mathrm{P} \$ \% O \rho_{12} r_{2}\right) \\
& \hat{d}_{2}{ }^{(1)}=\operatorname{Sgn}\left(r_{2} \mathrm{P} \$ \% O \rho_{21} r_{1}\right)
\end{aligned}
$$

Here $\rho_{12}$ and $\rho_{21}$ is the cross correlations between user, $r_{1}$ and $r_{2}$ are received signals at input of first stage and $d_{1} \& d_{2}$ are output of stages of detectors. The outputs of the next stages find as similar to equations (8) and (9).

\section{B. Decision-feedback detectors}

These detectors are similar to decision feedback equalizers employed in single user channels with ISI [7]. In decision feedback multiuser detection, users are ranked in order of decreasing received power levels. Previous decisions are then used together with current statistics to estimate the current output bits. In each time frame 
decisions are made in the order of decreasing user's strength, i.e., the stronger users make decisions first, allowing the weaker user to utilize these decisions. The diagram of the Decorrelating decision- feedback detector for synchronous CDMAis shown in Fig. 4.

At the output of the sorter, users are ranked according to their powers, so that stronger user is ranked first, and the weakest is ranked last. Decision feedback can be characterized by two matrix transformation, first a whitening feed forward filter followed by sorter that operates on the matched filter outputs and second feedback filter fed by the vector of previously made bit decisions.

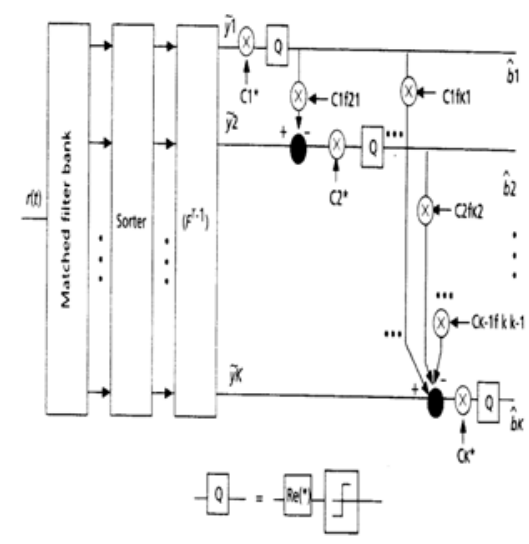

Fig. 4. The Decorrelating decisions feedback detector

The noise whitening filter is realized by Cholesky factorization of the correlation matrix $R$, which yields a resulting MAI matrix that is lower triangular. Consequently, at the out put of the whitening filter, the signal of the k'th strongest user $y_{k}$ is given by:

$y_{k}=$ desired signal + MAl due to stronger user $(1,2, k-1)+$ noise

In particular, the signal of the strongest user $y$ is not corrupted by MAI, and can be demodulated first. This decision is then used to subtract MAl from the signal of the second user, and so on.

\section{Subtractive interference cancellation}

The basic principle of these detectors is the creation at the receiver of separate estimates of the MAI contributed by each user in order to subtract out some or all of the MAI seen by each user. Such detectors are often implemented with multiple stages, where the expectation is that the decisions will improve at the output of the successive stages. The bit decision used to estimate the MAI can be hard or soft. The soft decision approach uses soft data estimates for the joint estimation of the data and amplitudes and easier to implement. The hard decision approach [8] feeds back a bit decision and is nonlinear and require reliable estimates of the MAI. To facilitate exposition, such detectors can be broken down into two categories, although the categories are not disjoint and particular realizations of sub-optimal detectors may use combination of these categories. First type is successive interference cancellation (SIC) and second is parallel interference cancellation (PIC).the details of such detectors are as follows.

Successive interference cancellation (SIC): The Successive Interference Cancellation (SIC) detector takes a serial approach to interference cancellation. Each stage of this detector decisions, regenerates and cancels out one direct sequence user from the received signal, so that the remaining users see less MAl in the next stage. It is intuitively clear that the users must be demodulated in order of decreasing power. The SIC detector is thus preceded by a stage which ranks users in descending order of received power. The result is that the strongest user will not benefit from any MAI reduction; the weakest user will however see a huge reduction in its MAI. The detector comprises of $\mathrm{K}$ stages in cascade, where $K$ is the number of active users in the system. At the k'th stage, a decision is made on the transmitted bit of the k'th user and its signal is regenerated and subtracted from the received signal. A "cleaner" version of the signal is thus obtained, which is then used to demodulate the subsequent user. The SIC detector offers significant performance improvements, especially when there is large disparity amongst received power levels. The detector is easily realizable in hardware. The performance is however extremely sensitive to initial bit estimates and to power level estimates. Also, the sorting operation which needs to be performed at the beginning of each bit interval leads to increase in complexity.

Parallel interference cancellation (PIC): In contrast to the SIC, the PIC detector estimates and subtracts out all of the MAI for each user in parallel. The initial bit estimates are typically derived from a conventional detector; however use of decorrelating or minimum mean square error (MMSE) detectors has also been suggested in literature. The estimated bits are scaled by received power level estimates and re-spread by signature codes. A partial summer then sums up all but one of the signals at each of the outputs, which creates the complete MAl estimate for each user.

This process can be repeated for multiple stages. Each stage takes as input the bit estimates of the preceding stage and produces a new set of estimates at its output. Like the SIC, the performance of this detector is severely affected by incorrect initial bit estimates and erroneous received power estimates. The parallel interference cancellation (PIC) multistage detector performs better than successive interference cancellation (SIC) in a power controlled channel. 


\section{SIMULATION AND PERFORMANCE CRITERIA}

The non-linear suboptimal multiuser detectors for CDMA communication described in section $V$ was simulated in MATLAB, particularly popular in wireless communication. In simulation process, mainly one parameter was varied, the signal to noise ratio (SNR) for different mentioned detector. The performance criteria for evolution of multistage multiuser detector is bit error rate (BER) has been taken for this paper. The reduced bit error rate shows reduced multiple access interference, thus the capacity of the system will increase. The parameters were used for simulation as follows.

\section{TABLE 1}

Simulation Parameters For Non-linear Multiuser Detector

\begin{tabular}{|cc|}
\hline Parameter & Value \\
\hline Window length (L) & 128 \\
Spreading gain (N) & 32 \\
Signal to interference noise & $0 \mathrm{~dB}$ \\
ratio (SINR) & 3 \\
Number of paths (P) & $3,5,7,8,9,10,12,14 \& 16$ \\
Signal to noise ratio (SNR) & $\mathrm{dB}$ \\
Number of user & 20 \\
Number of PIC stages & 4 \\
\hline
\end{tabular}

\section{SIMULATION RESULTS}

The bit error rate $(B E R)$ at different signal to noise ratio (SNR) was obtained for various nonlinear detectors as given in Table 2.

\section{TABLE 2}

Value of BER for different multiuser detectors

\begin{tabular}{|cccc|}
\hline SNR & $\begin{array}{c}\text { Decision } \\
\text { feedback } \\
\text { detector } \\
\text { (dB) }\end{array}$ & $\begin{array}{c}\text { SIC } \\
\text { detector }\end{array}$ & $\begin{array}{c}\text { Multistage } \\
\text { PIC detector } \\
\text { (BER) }\end{array}$ \\
\hline 3 & 0.1710 & (BER) & \\
5 & 0.1134 & 0.1528 & 0.1470 \\
7 & 0.0875 & 0.0654 & 0.0891 \\
8 & 0.0765 & 0.0598 & 0.0487 \\
9 & 0.0548 & 0.0385 & 0.0234 \\
10 & 0.0402 & 0.0224 & 0.0154 \\
12 & 0.0295 & 0.0106 & 0.0064 \\
14 & 0.0197 & 0.0087 & 0.0024 \\
16 & 0.0107 & 0.0032 & 0.0008 \\
\hline
\end{tabular}

The bit error rate at different signal to noise ratio for different multiuser detector was also shown in graph form in Fig. 5.

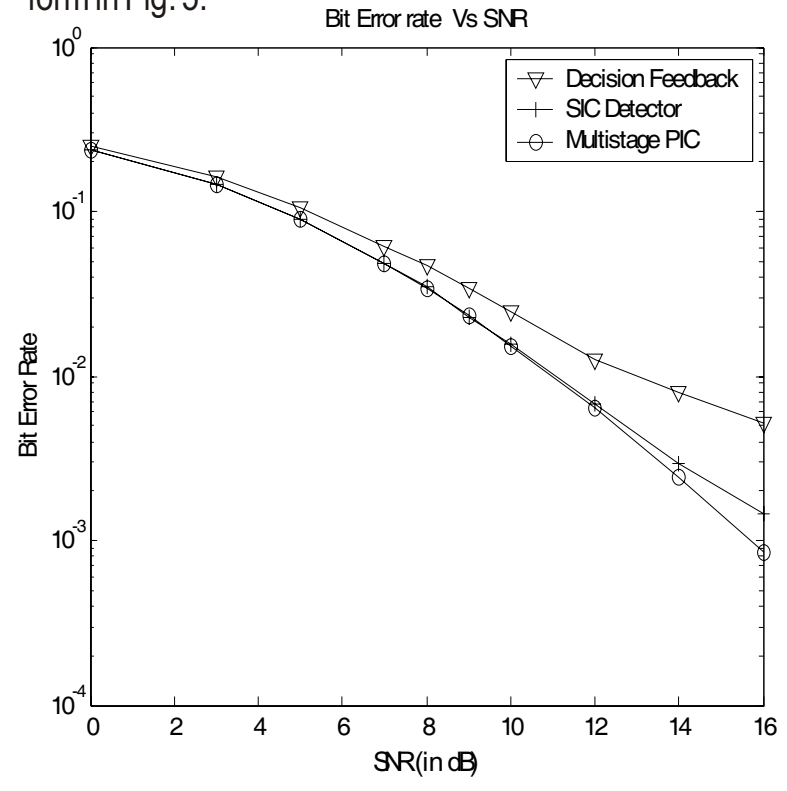

Fig.5. SNR Vs BER for different NMUD

The BER decreases gradually beyond $2 \mathrm{~dB}$ SNR. The multistage parallel interference cancellation detector gives the best BER for a given SNR among the described detectors.

\section{CONCLUSION}

In this paper, we have provided simulation results of three nonlinear multiuser detectors for DS-CDMA systems. The results shown that for perfect power control, the multistage parallel interference cancellation detector outperform among the described detectors. When channel estimation is not an issue, nonlinear multiuser detectors provide the best performance. However, if channel estimation performance is adequate and the desired BER's are in the range of $10^{-2}$, then the performances of the detectors are nearly identical. Between the two cancellation schemes, successive is overall less computationally intensive and easy to implementation but parallel scheme is more flexible and gives lower bit error rate in comparison to successive scheme. The major disadvantage of nonlinear detectors is their dependence on reliable estimation of the received amplitudes. Imperfect amplitude estimation may significantly reduces or even reverse the gain to be had from using these detectors. Result shows that if nonlinear multiuser detectors are commercially feasible, can greatly increase the attractiveness of CDMA for mobile and personal communication system. 


\section{ACKNOWLEDGMENT}

The authors acknowledge the pioneer work done by Prof. S. Verdu, Prof. S. Moshavi and many others researchers in this area.

\section{REFERENCES}

[1] Lokesh Tharani and Dr. R.P.Yadav, 2007 "Performance evaluation of multistage multiuser detector for asynchronous DS-CDMA system" IEEE Conference Proceeding of International Conference on Computational Intelligence and Multimedia Applications, Vol.-IV , page no. 316-321, December 2007, Sivkasi, India.

[2] Sergio Verdu, "Multi-user detection", Cambridge University Press. ISBN: 0521-59373-5.

[3] Hallen, J. Holtzman, and Z. Zvonar,April 1995 "Multi-user detection for CDMA systems", IEEE Personal Communications Magazine.

[4] Moshavi, October, 1996 "Multi-user detection for DSCDMA communications", IEEE communications magazine, Vol. 34, No. 10, page no. 124-136.
[5] H. V. Poor, S. Verdu,Jan. 1988. "Single-user detectors for multiuser channels," IEEE Trans. Comm. Vol. 36, no. 1, pp. 50-60.

[6] Lupas., S. Verdu, April 1990 "Near-far resistance of multiuser detectors in asynchronous channels," , IEEE Trans. Comm. Vol. 38, no. 4, pp. 496-508.

[7] J. G. Prokis, 2001 "Digital communication", forth edition, McGraw-Hill International .

[8] Z. Afzal Uzmi, September, 2002 "Simplified multiuser detection for CDMAsystems", Ph.D., Thesis.

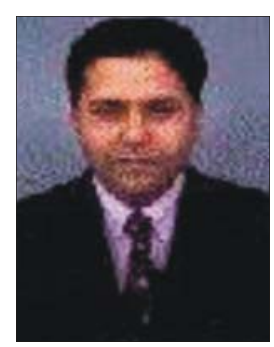

Lokesh Tharani has done BE (Hons.) from Govt. Engineering College Kota (Now UCE) in 1999 and completed M.Tech. (Hons.) in Electronics \& Communication Engineering from Malaviya National Institute of Technology (MNIT), Jaipur in 2003. He is also pursuing his Ph.D. in CDMA technology from MNIT, Jaipur. He has a teaching experience of more than 9 years and has published several papers in national \& international International Journals and conferences. 